\title{
Dinâmica da matéria orgânica e da biomassa microbiana em solo submetido a diferentes sistemas de manejo na Amazônia Ocidental
}

\author{
Adônis Moreira(1) e Eurípedes Malavolta ${ }^{(2)}$
}

(1)Embrapa Amazônia Ocidental, Caixa Postal 319, CEP 69011-970 Manaus, AM. E-mail: adonis@cpaa.embrapa.br (2)Universidade de São
Paulo, Centro de Energia Nuclear na Agricultura, Caixa Postal 96, CEP 13416-970 Piracicaba, SP. E-mail: mala@cena.usp.br

Resumo - O objetivo deste trabalho foi avaliar as alterações na atividade da biomassa microbiana como um indicador da dinâmica de $\mathrm{C}$ e $\mathrm{N}$ em solo submetido à sucessão de cobertura vegetal e de manejo na Amazônia Ocidental. Os trabalhos foram realizados em duas cronosseqüências: CA - sucessão floresta primária e cupuaçuzal (Theobroma grandiflorum) de três anos - e CB - sucessão floresta primária, pastagem de Brachiaria humidicola de oito anos e cupuaçuzal de três anos. A sucessão floresta primária-pastagem-cupuaçuzal afeta negativamente o estoque de $\mathrm{C}$ do solo, com diminuição significativa da matéria orgânica e do C da biomassa microbiana do solo, ao passo que na sucessão floresta primária-cupuaçuzal ocorre diminuição apenas do C da biomassa microbiana. A floresta primária apresenta menor quociente metabólico e maior relação $\mathrm{C} / \mathrm{N}$ da biomassa, o que resulta em menor perda de carbono. $\mathrm{O} \mathrm{N}$ da biomassa microbiana na camada de $0-10 \mathrm{~cm}$, independentemente do manejo adotado, aumenta significativamente na área de cupuaçuzal, ao passo que o $\mathrm{N}$ total diminui. A concentração de amônio no solo diminui com a profundidade, de modo oposto ao verificado com o nitrato.

Termos para indexação: Theobroma grandiflorum, floresta primária, carbono, nitrogênio, respiração basal, quociente metabólico.

\section{Dynamic of organic matter and microbial biomass in a soil under different management systems in the Western Amazon}

\begin{abstract}
The objective of this work was to evaluate the alterations of the microbial biomass activity as an indicator of $\mathrm{C}$ and $\mathrm{N}$ dynamics in a soil submitted to the succession of plant cover and management in the Western Amazon. The work was carried out in two chronosequences: CA - a succession from primary forest to a three years old cupuaçu (Theobroma grandiflorum) plot - and CB - a succession from primary forest to an eight years old Brachiaria humidicola pasture and a three years old cupuaçu plot. The succession primary forest-pasture-cupuaçu affects negatively the stock of the soil carbon, with significant decrease of organic matter and $\mathrm{C}$ of the soil microbial biomass. The primary forest presents lower metabolic quotient and higher $\mathrm{C} / \mathrm{N}$ ratio of the biomass, which results in less carbon loss. The $\mathrm{N}$ of the microbial biomass found in the $0-10 \mathrm{~cm}$ layer irrespective of the management adopted, increases significantly in the cupuaçu plot and the total $\mathrm{N}$ decreases. Ammonium concentration decreases according to soil depth, but this does not occur with nitrate.
\end{abstract}

Index terms: Theobroma grandiflorum, forest, nitrogen, carbon, basal respiration, metabolic quotient.

\section{Introdução}

A Bacia Amazônica abrange uma área no território brasileiro de aproximadamente 4,5 milhões de $\mathrm{km}^{2}$, dos quais $75 \%$ são solos de baixa fertilidade (Sanchez et al., 1982), o que consiste num problema para qualquer tipo de cultivo, principalmente quando se considera a escassez de infra-estrutura da região no que se refere à disponibilidade de insumos agrícolas.

O uso da terra consiste no desmatamento, remoção da madeira com expressividade econômica e a posterior utilização de queimadas. A seguir, pode ocorrer a introdução de culturas anuais, perenes ou formação de pastagens (Andreux \& Cerri, 1989). Segundo Diez et al. (1991), as mudanças no conteúdo de nutrientes e nas propriedades físicas do solo têm sido relacionadas com diferentes formas de cultivo. Vários estudos relataram aumento no $\mathrm{pH}$ do solo, no teor de cátions trocáveis e redução da acidez trocável decorrentes do desmatamento e queima da floresta natural (Martins et al., 1991). O desmatamento também afeta significativamente o conteúdo de $\mathrm{C}$ e $\mathrm{N}$ do solo (Martins et al., 1991; Feigl, 1994). 
Por esse motivo, a produtividade dos ecossistemas naturais e de agrossistemas introduzidos, e raramente fertilizados, depende da reciclagem dos nutrientes minerais contidos na serapilheira das plantas e da matéria orgânica do solo. O balanço entre produtividade primária e perdas por lixiviação, erosão, volatilização e exportação de nutrientes pelas culturas reflete sua sustentabilidade agronômica (Fearnside, 1993).

A remoção da floresta para fins agrícolas causa uma quebra nos ciclos do carbono e dos nutrientes (Malavolta, 1987), os quais operam graças à entrada fotossintética do gás carbônico e à decomposição acelerada e contínua da matéria orgânica do solo (MO), realizada pelos microorganismos. Nesse contexto, a alteração da qualidade e da quantidade de matéria orgânica afeta a atividade da biomassa microbiana.

Os efeitos das mudanças do uso da terra sobre a quantidade e qualidade da MO sob cultivos itinerantes ou pastagens ainda não são bem compreendidos, embora sejam reconhecidamente benéficos para a fertilidade do solo e além disso, o manejo adequado pode ser visto como um promotor da sustentabilidade da agricultura nos trópicos (Cerri et al., 1985).

Os microrganismos, mesmo representando uma pequena fração do total de matéria orgânica do solo, são responsáveis pelos processos de mineralização, contendo uma quantidade considerável de nutrientes $(\mathrm{N}, \mathrm{P}, \mathrm{S}, \mathrm{Zn}$ e $\mathrm{Cu})$ potencialmente disponíveis para as plantas (Jenkinson, 1988). A população microbiana tem sido considerada um indicador sensível das alterações no estoque de MO decorrentes do uso da terra (Sparling \& Ross, 1993).

Apesar de sua importância, existe um número limitado de estudos sobre efeito do desflorestamento na MO, biomassa microbiana e população microbiana (Luizão et al., 1992; Feigl, 1994) em solos da Floresta Amazônica brasileira. A biomassa microbiana do solo, definida por Jenkinson \& Ladd (1981) como o componente microbiano vivo, é composta de fungos, bactérias, microfauna e algas, com participação fundamental nos ciclos biogeoquímicos de interesse para a produtividade agrícola.

O objetivo deste trabalho foi avaliar as alterações na atividade da biomassa microbiana como indicador da dinâmica de $\mathrm{C}$ e $\mathrm{N}$ em solo submetido à sucessão de cobertura vegetal e de manejo na Amazônia Ocidental.

\section{Material e Métodos}

O trabalho foi realizado em Latossolo Amarelo distrófico (Embrapa, 1999), cujas características quími- cas e físicas são apresentadas na Tabela 1. O local do trabalho encontra-se nas coordenadas geográficas $3^{\circ} 8^{\prime} 25^{\prime \prime}$ LS e 5952' LW, Município de Manaus, AM. O clima é tropical úmido, tipo Afi, segundo a classificação de Köppen, com chuvas relativamente abundantes durante todo o ano (média de $2.250 \mathrm{~mm}$ ), e a quantidade no mês de menor precipitação é sempre superior a $60 \mathrm{~mm}$. A temperatura média da região é de aproximadamente $26^{\circ} \mathrm{C}$ (Vieira \& Santos, 1987).

Foram estudadas duas cronossequiências: $\mathrm{CA}$ - sucessão floresta primária e cupuaçuzal [Theobroma grandiflorum (Wild. ex Spreng.) Schum.] de três anos, na qual ocorreu somente retirada da madeira, abertura das covas, adubação e plantio (manejo 1) -, e CB sucessão floresta primária e cupuaçuzal de três anos, plantado em área de pastagem de Brachiaria humidicola (Rendle) Schw., mantida por oito anos, com posterior abertura das covas, adubação e plantio (manejo 2). O manejo inicial das duas áreas consistiu no corte e remoção da madeira, enleiramento e queima do material vegetal.

Nas duas áreas foram coletadas amostras de solo nas profundidades de $0-10 \mathrm{~cm}, 10-20 \mathrm{~cm}, 20-40 \mathrm{~cm}$ e 40-60 cm, em transecto, 30 pontos distando $10 \mathrm{~m}$ entre eles, perfazendo no total $300 \mathrm{~m}$. O delineamento estatístico utilizado foi de blocos casualisados com parcelas subdivididas (posição da amostragem) em dois tratamentos, ou seja, área de cultivo (cupuaçu) e floresta primária (controle), com três repetições. Nas duas cronosseqüências usaram-se os critérios estatísticos descritos por Longo (1999), que estabelecem que as repetições sejam tomadas ao longo da própria transecção, sendo que a soma de cada três pontos seqüenciais de amostragem de cada linha constituiu uma parcela.

Nas amostras de solo coletadas nas quatro profundidades, foram analisados os teores de $\mathrm{MO}$ e $\mathrm{N}$ total, por digestão sulfúrica, $\mathrm{NH}_{4}{ }^{+}$e $\mathrm{NO}_{3}{ }^{-}$extraídos com solução de $\mathrm{KCl}$ 1,0 mol L-1. A determinação da biomassa microbiana de $\mathrm{C}$ e $\mathrm{N}$ foi realizada somente na profundidade de 0-10 cm, pelo método de irradiação e extração (Ferreira et al., 1999). No cálculo do carbono da biomassa microbiana, utilizou-se Kc de 0,45 e 0,54 para C e N, respectivamente (Balota et al., 1998).

Outro método utilizado foi a determinação indireta mediante a respiração microbiana com IRGA (Infra Red Gas Analyzer), conforme Anderson \& Domsch (1978). A respiração induzida foi transformada em biomassa microbiana pela fórmula: 
Biomassa em mg C kg-1 de solo = (respiração em $\left.\mu \mathrm{L} \mathrm{CO} \min ^{-1} \mathrm{~g}^{-1} \times 40,04\right)+0,37$.

O fluxo de $\mathrm{CO}_{2}\left(\mathrm{~mL} \mathrm{~min}^{-1}\right)$ foi determinado no IRGA nas amostras induzidas com $0,24 \mathrm{~g}$ de glicose $+0,50 \mathrm{~g}$ de talco inerte (Anderson \& Domsch, 1978). A respiração basal ( $\mu \mathrm{g} \mathrm{g}^{-1}$ dia $^{-1}$ de C-CO $\mathrm{CO}_{2}$ no solo) foi determinada nas mesmas amostras antes da indução, refletindo a atividade microbiana.

$\mathrm{O}$ quociente metabólico $\left(\mathrm{qCO}_{2}\right)$, que representa a quantidade de $\mathrm{CO}_{2}$ liberado por unidade de biomassa microbiana, foi obtido pela divisão da respiração basal pelo carbono da biomassa microbiana do solo e os valores foram expressos em $\left(\mu \mathrm{g} \mathrm{CO}_{2} / \mathrm{mg} \mathrm{Cmin} \mathrm{h}^{-1}\right) \times 10^{-4}$.

Após a determinação da atividade microbiana, o solo foi secado ao ar e peneirado, exceto nas análises de densidade e porosidade do solo, que foram realizadas em amostras indeformadas (Embrapa, 1997). Posteriormente, foram determinados os atributos químicos e físicos do solo (Tabela 1), segundo Mehlich (1984), Embrapa (1997) e Raij et al. (2001). As análises de variância, o teste $\mathrm{F}$, teste de comparação de contraste entre médias (Tukey a 5\% de probabilidade) e as correlações foram feitas de acordo com Pimentel-Gomes (1990).

\section{Resultados e Discussão}

A conversão da floresta primária em cupuaçuzal acarretou alterações nas características químicas e físicas do solo (Tabela 1). A mudança da cobertura vegetal e o tipo de manejo resultaram em aumentos significativos do $\mathrm{pH}, \mathrm{P}, \mathrm{K}, \mathrm{Ca}, \mathrm{Mg}$ e da densidade do solo e diminuição da concentração do $\mathrm{Al}$ trocável. Essas alterações foram decorrentes, principalmente, da prática da queima na abertura das áreas para o plantio, a qual introduz ao sistema grandes quantidades de cinzas provenientes da queima da floresta primária na camada superficial do solo, aumentando, muitas vezes, a fertilidade (Fernandes, 1999).

Apesar do aumento da fertilidade, o solo apresentou, nos três ecossistemas (floresta primária, manejo $1 \mathrm{e}$

Tabela 1. Atributos químicos e físicos do solo da camada de $0-10 \mathrm{~cm}$ de profundidade das três coberturas vegetais ${ }^{(1)}$.

\begin{tabular}{|c|c|c|c|c|}
\hline \multirow[t]{2}{*}{ Atributos } & \multicolumn{2}{|c|}{ Cronosseqüência $\mathrm{A}$} & \multicolumn{2}{|c|}{ Cronosseqüência B } \\
\hline & Floresta primária & Manejo $1^{(2)}$ & Floresta primária & Manejo $2^{(3)}$ \\
\hline $\mathrm{pH}$ em $\mathrm{H}_{2} \mathrm{O}$ & $4,28 \mathrm{~B}$ & $4,40 \mathrm{~A}$ & $4,46 \mathrm{a}$ & $4,77 \mathrm{a}$ \\
\hline $\mathrm{pH}$ em KCl & $3,85 \mathrm{~B}$ & $3,91 \mathrm{~A}$ & $3,97 \mathrm{a}$ & $4,11 \mathrm{a}$ \\
\hline $\mathrm{pH}$ em $\mathrm{CaCl}_{2}$ & $3,43 \mathrm{~B}$ & $3,59 \mathrm{~A}$ & $3,51 \mathrm{~b}$ & $3,71 \mathrm{a}$ \\
\hline$\Delta \mathrm{pH}$ & $-0,44 \mathrm{~A}$ & $-0,49 \mathrm{~A}$ & $-0,48 b$ & $-0,65 \mathrm{a}$ \\
\hline $\mathrm{P}-$ Mehlich $1\left(\mathrm{mg} \mathrm{dm}^{-3}\right)$ & 2,94B & $5,13 \mathrm{~A}$ & $1,95 b$ & $3,54 \mathrm{a}$ \\
\hline $\mathrm{P}-\operatorname{resina}\left(\mathrm{mg} \mathrm{dm}^{-3}\right)$ & $9,80 \mathrm{~B}$ & $16,77 \mathrm{~A}$ & $10,25 b$ & $11,37 \mathrm{a}$ \\
\hline $\mathrm{P}-$ Mehlich $3\left(\mathrm{mg} \mathrm{dm}^{-3}\right)$ & $1,78 \mathrm{~B}$ & $3,91 \mathrm{~A}$ & $3,10 \mathrm{~b}$ & $4,02 \mathrm{a}$ \\
\hline $\mathrm{K}-$ Mehlich $1\left(\mathrm{cmol}_{\mathrm{c}} \mathrm{dm}^{-3}\right)$ & $0,06 \mathrm{~B}$ & $0,12 \mathrm{~A}$ & $0,06 \mathrm{~b}$ & $0,11 \mathrm{a}$ \\
\hline $\mathrm{K}-\operatorname{resina}\left(\mathrm{cmol}_{\mathrm{c}} \mathrm{dm}^{-3}\right)$ & $0,06 \mathrm{~B}$ & $0,13 \mathrm{~A}$ & $0,12 b$ & $0,17 \mathrm{a}$ \\
\hline $\mathrm{K}-$ Mehlich $3\left(\mathrm{cmol}_{\mathrm{c}} \mathrm{dm}^{-3}\right)$ & $0,07 \mathrm{~B}$ & $0,13 \mathrm{~A}$ & $0,08 b$ & $0,14 \mathrm{a}$ \\
\hline $\mathrm{Ca}-$ Mehlich $1\left(\mathrm{cmol}_{\mathrm{c}} \mathrm{dm}^{-3}\right)$ & $0,19 \mathrm{~B}$ & $0,54 \mathrm{~A}$ & $0,41 \mathrm{a}$ & $0,48 \mathrm{a}$ \\
\hline $\mathrm{Ca}-\operatorname{resina}\left(\mathrm{cmol}_{\mathrm{c}} \mathrm{dm}^{-3}\right)$ & $0,20 \mathrm{~B}$ & $0,61 \mathrm{~A}$ & $0,30 \mathrm{a}$ & $0,40 \mathrm{a}$ \\
\hline $\mathrm{Ca}-$ Mehlich $3\left(\mathrm{cmol}_{\mathrm{c}} \mathrm{dm}^{-3}\right)$ & $0,37 \mathrm{~B}$ & $0,55 \mathrm{~A}$ & $0,71 \mathrm{~b}$ & $1,17 \mathrm{a}$ \\
\hline $\mathrm{Mg}-$ Mehlich $1\left(\mathrm{cmol}_{\mathrm{c}}^{\mathrm{c}} \mathrm{dm}^{-3}\right)$ & $0,19 \mathrm{~B}$ & $0,36 \mathrm{~A}$ & $0,14 \mathrm{~b}$ & $0,26 \mathrm{a}$ \\
\hline $\mathrm{Mg}-\operatorname{resina}\left(\mathrm{cmol}_{\mathrm{c}} \mathrm{dm}^{-3}\right)$ & $0,25 \mathrm{~B}$ & $0,30 \mathrm{~A}$ & $0,25 \mathrm{a}$ & $0,30 \mathrm{a}$ \\
\hline $\mathrm{Mg}-\operatorname{Mehlich} 3\left(\mathrm{cmol}_{\mathrm{c}} \mathrm{dm}^{-3}\right)$ & $0,15 \mathrm{~B}$ & $0,22 \mathrm{~A}$ & $0,15 b$ & $0,38 \mathrm{a}$ \\
\hline $\mathrm{Al}-\mathrm{KCl}\left(\mathrm{cmol}_{\mathrm{c}} \mathrm{dm}^{-3}\right)$ & $1,60 \mathrm{~A}$ & $1,29 \mathrm{~B}$ & $1,50 \mathrm{a}$ & $0,99 b$ \\
\hline $\mathrm{H}+\mathrm{Al}\left(\mathrm{cmol}_{\mathrm{c}} \mathrm{dm}^{-3}\right)$ & $8,16 \mathrm{~A}$ & $8,27 \mathrm{~A}$ & $8,11 \mathrm{a}$ & $6,34 b$ \\
\hline $\mathrm{m}(\%)$ & $82,81 \mathrm{~A}$ & $62,25 \mathrm{~B}$ & $75,49 \mathrm{a}$ & $52,28 \mathrm{~b}$ \\
\hline CTC $\left(\mathrm{cmol}_{\mathrm{c}} \mathrm{dm}^{-3}\right)-$ Mehlich 1 & $8,60 \mathrm{~B}$ & $9,29 \mathrm{~A}$ & $8,72 \mathrm{a}$ & $7,19 b$ \\
\hline $\mathrm{V}(\%)$ & $3,95 \mathrm{~B}$ & $9,10 \mathrm{~A}$ & $5,93 b$ & $13,92 \mathrm{a}$ \\
\hline $\operatorname{Argila}\left(\mathrm{g} \mathrm{kg}^{-1}\right)$ & $695,70 \mathrm{~A}$ & $711,63 \mathrm{~A}$ & $710,79 a$ & $725,44 a$ \\
\hline Densidade $\left(\mathrm{g} \mathrm{cm}^{-3}\right)$ & 0,79B & $0,92 \mathrm{~A}$ & $0,79 b$ & $1,02 \mathrm{a}$ \\
\hline Porosidade $\left(\mathrm{m}^{3} \mathrm{~m}^{-3}\right)$ & $0,71 \mathrm{~A}$ & $0,65 \mathrm{~B}$ & $0,69 \mathrm{a}$ & $0,60 \mathrm{~b}$ \\
\hline
\end{tabular}

${ }^{(1)}$ Médias seguidas por letras distintas maiúsculas (cronosseqüência A) e minúsculas (cronosseqüência B) na mesma linha diferem entre si a 5\% de probabilidade pelo teste de Tukey. ${ }^{(2)}$ Manejo 1: cupuaçuzal de três anos, cuja implantação não utilizou implementos agrícolas, havendo somente a retirada da madeira, abertura e adubação das covas e plantio. ${ }^{(3)}$ Manejo 2: cupuaçuzal de três anos, instalado sob área de pastagem de Brachiaria humidicula (oito anos), com posterior adubação das covas e plantio. 
manejo 2), baixos teores de nutrientes. Segundo Carvalho (1986), esses baixos valores podem ser considerados como um índice da evolução atingida pelo solo. O mesmo autor salientou que esses valores são atribuídos ao rápido intemperismo do material de origem, que apresenta baixos teores de bases, com pouco ou nenhum material primário. Esses baixos valores confirmaram o alto grau de evolução do solo (Latossolo Amarelo distrófico), com o hidrogênio e alumínio prevalecendo no complexo trocável, em substituição às bases (Falesi et al., 1969).

Entre as duas cronosseqüências, houve variação no conteúdo de nutrientes obtidos pelos extratores Mehlich 1, Mehlich 3 e resina (Tabela 1). Na CA, os maiores teores de $\mathrm{P}$ disponível foram obtidos com os extratores Mehlich 1 e resina, e o inverso ocorreu com o extrator Mehlich 3. A CB apresentou os maiores teores de K disponível (extratores Mehlich 1 e resina). A floresta primária estudada na $\mathrm{CB}$ apresentou o maior conteúdo de Ca disponível, independentemente do extrator utilizado. Estes resultados mostram a complexidade em se definir qual o extrator mais adequado na análise de rotina.

Os teores de $\mathrm{N}$ total nas duas cronossequiências, corrigidos pelo teor de argila (Feller et al., 1991), indicaram que as mudanças na cobertura vegetal promovem uma diminuição significativa nos teores deste elemento no solo, com redução de 13,5\% (0-10 cm), 4,3\% (10-20 cm), 4,7\%
(20-40 cm) e 7,6\% (40-60 cm) na cronosseqüência A, e de $8,2 \%(0-10 \mathrm{~cm}), 11,0 \%(10-20 \mathrm{~cm}), 20,9 \%$ $(20-40 \mathrm{~cm})$ e $23,1 \%(40-60 \mathrm{~cm})$ na B (Tabela 2). Essa diminuição deve-se, principalmente, à redução na quantidade de MO. A diminuição no conteúdo de $\mathrm{N}$ está associada a mudanças significativas na estrutura do solo, afetando sua atividade biológica.

Nas duas cronossequiências, independentemente da cobertura vegetal, a camada de $0-10 \mathrm{~cm}$ apresentou os maiores teores de $\mathrm{N}$, com diminuição significativa nas mais profundas (Tabela 2). Estes resultados corroboram os de Cerri (1989), que em solos da Bacia Amazônica, observou que a maior parte do $\mathrm{N}$ total encontra-se estocada na camada de $0-20 \mathrm{~cm}$ de profundidade. Essas variações nos teores de $\mathrm{N}$ total ocorrem em razão de uma série de fatores, como clima, $\mathrm{pH}$, atividade microbiana, propriedades físicas do solo, manejo, vegetação e material de origem (Jenny, 1941; Longo, 1999).

Os teores de matéria orgânica (MO) apresentaram, nas duas cronosseqüências, comportamento similar ao do $\mathrm{N}$ total (Tabela 2). Os maiores valores foram encontrados na camada de $0-10 \mathrm{~cm}$, havendo diminuição significativa nas camadas inferiores. A redução média nas camadas amostradas foi de $24,0 \%$ da camada de 0-10 cm para $10-20 \mathrm{~cm}, 22,6 \%$ da camada de $10-20 \mathrm{~cm}$ para $20-40 \mathrm{~cm}$ e $13,6 \%$ da camada de $20-40 \mathrm{~cm}$ para $40-60 \mathrm{~cm}$.

Tabela 2. Matéria orgânica, nitrogênio total, amônio, nitrato, nitrogênio mineral e relação $\mathrm{C} / \mathrm{N}$ nas camadas de $0-10 \mathrm{~cm}$, 10-20 cm, 20-40 cm e 40-60 cm de profundidade ${ }^{(1)}$.

\begin{tabular}{|c|c|c|c|c|c|c|c|c|c|c|c|c|}
\hline Profundidade & $\begin{array}{c}\text { Floresta } \\
\text { MO }\end{array}$ & $\begin{array}{c}\text { Manejo } \\
\text { MO }\end{array}$ & $\begin{array}{l}\text { Floresta } \\
\mathrm{N} \text { total } \\
\mathrm{kg}^{-1} \text { )------ }\end{array}$ & $\begin{array}{l}\text { Manejo } \\
\text { N total }\end{array}$ & $\begin{array}{l}\text { Floresta } \\
\mathrm{N}-\mathrm{NH}_{4}^{+}\end{array}$ & $\begin{array}{l}\text { Manejo } \\
\mathrm{N}-\mathrm{NH}_{4}^{+}\end{array}$ & $\begin{array}{c}\text { Floresta } \\
\mathrm{N}^{-} \mathrm{NO}_{3}^{-}\end{array}$ & $\begin{array}{l}\text { Manejo } \\
{\mathrm{N}-\mathrm{NO}_{3}^{-}}^{-} \\
\left.\mathrm{g} \mathrm{kg}^{-1}\right)-\end{array}$ & $\begin{array}{c}\text { Floresta } \\
\mathrm{N} \text { mineral }\end{array}$ & $\begin{array}{c}\text { Manejo } \\
\text { N mineral }\end{array}$ & $\begin{array}{c}\text { Floresta } \\
\text { C/N }\end{array}$ & $\begin{array}{c}\text { Manejo } \\
\mathrm{C} / \mathrm{N}\end{array}$ \\
\hline & \multicolumn{12}{|c|}{ Cronosseqüência $\mathrm{A}^{(2)}$} \\
\hline $0-10$ & $42,77 \mathrm{aA}$ & $41,89 \mathrm{aA}$ & $1,87 \mathrm{aA}$ & $1,66 \mathrm{aB}$ & $2,4 \mathrm{aA}$ & $1,8 \mathrm{aA}$ & $0,5 \mathrm{aB}$ & $3,9 \mathrm{aA}$ & $2,9 \mathrm{aB}$ & $5,7 \mathrm{aA}$ & $13,27 \mathrm{aB}$ & $14,64 \mathrm{aA}$ \\
\hline $11-20$ & $31,04 \mathrm{bA}$ & $31,43 \mathrm{bA}$ & $1,34 \mathrm{bA}$ & $1,35 \mathrm{bA}$ & $1,8 \mathrm{abA}$ & $1,4 \mathrm{abA}$ & $0,4 \mathrm{aB}$ & $3,6 \mathrm{abA}$ & $2,2 \mathrm{abB}$ & $5,0 \mathrm{aA}$ & $13,44 \mathrm{aA}$ & $13,50 \mathrm{bA}$ \\
\hline $21-40$ & $23,50 \mathrm{cA}$ & $23,58 \mathrm{cA}$ & $1,07 \mathrm{cA}$ & $1,03 \mathrm{cA}$ & $1,2 \mathrm{bcA}$ & $1,1 \mathrm{bcA}$ & $0,3 \mathrm{aB}$ & $2,7 \mathrm{bA}$ & $1,5 \mathrm{bcB}$ & $3,8 \mathrm{bA}$ & $12,73 \mathrm{bB}$ & $13,28 \mathrm{bA}$ \\
\hline $41-60$ & $19,65 \mathrm{dA}$ & $18,96 \mathrm{dA}$ & $0,97 \mathrm{dA}$ & $0,90 \mathrm{dA}$ & $0,7 \mathrm{cA}$ & $0,5 \mathrm{cA}$ & $0,2 \mathrm{aB}$ & $2,7 \mathrm{bA}$ & $0,9 \mathrm{cB}$ & $3,2 \mathrm{bA}$ & $11,90 \mathrm{cB}$ & $12,67 \mathrm{cA}$ \\
\hline $\mathrm{CV}(\%)$ & 17,74 & & 13,97 & & 15,44 & & 18,14 & & 22,21 & & 10,30 & \\
\hline \multicolumn{13}{|c|}{ Cronosseqüência B $^{(3)}$} \\
\hline $0-10$ & $47,70 \mathrm{aA}$ & $40,82 \mathrm{aB}$ & $1,83 \mathrm{aA}$ & $1,71 \mathrm{aB}$ & $2,2 \mathrm{aB}$ & $8,7 \mathrm{aA}$ & $0,5 \mathrm{aB}$ & $6,4 \mathrm{aA}$ & $2,7 \mathrm{aB}$ & $15,1 \mathrm{aA}$ & $15,12 \mathrm{abA}$ & $13,85 \mathrm{aB}$ \\
\hline $11-20$ & $37,57 \mathrm{bA}$ & $29,27 \mathrm{bA}$ & $1,37 \mathrm{bA}$ & $0,91 \mathrm{bA}$ & $2,3 \mathrm{aB}$ & $5,0 \mathrm{bA}$ & $0,4 \mathrm{aB}$ & $5,4 \mathrm{aA}$ & $2,7 \mathrm{aB}$ & $9,3 \mathrm{bA}$ & $15,90 \mathrm{aA}$ & $13,37 \mathrm{abB}$ \\
\hline $21-40$ & $28,27 \mathrm{cA}$ & $18,91 \mathrm{cB}$ & $1,08 \mathrm{cA}$ & $0,91 \mathrm{cB}$ & $1,0 \mathrm{bB}$ & $3,9 \mathrm{cA}$ & $0,3 \mathrm{aB}$ & $5,4 \mathrm{aA}$ & $1,3 \mathrm{bB}$ & $9,3 \mathrm{bA}$ & $15,19 \mathrm{abA}$ & $12,07 \mathrm{bcB}$ \\
\hline 41-60 & $23,41 \mathrm{dA}$ & $14,33 \mathrm{cB}$ & $0,94 \mathrm{cA}$ & $0,76 \mathrm{cB}$ & $0,5 \mathrm{bB}$ & $4,0 \mathrm{bcA}$ & $0,2 \mathrm{aB}$ & $6,4 \mathrm{aA}$ & $0,7 \mathrm{bB}$ & $10,4 \mathrm{bA}$ & $14,45 \mathrm{bA}$ & $10,93 \mathrm{cB}$ \\
\hline$\overline{\mathrm{CV}(\%)}$ & 24,09 & & 16,68 & & 19,47 & & 14,87 & & 21,15 & & 19,50 & \\
\hline
\end{tabular}

Pesq. agropec. bras., Brasília, v.39, n.11, p.1103-1110, nov. 2004 
A sucessão da floresta primária por áreas de cultivo acarretou diferenças significativas no teor de $\mathrm{MO}$ em todas as camadas na cronossequiência $\mathrm{B}$; porém, na cronossequiência $\mathrm{A}$, não houve diminuição estatisticamente significativa da transecção na camada $0-10 \mathrm{~cm}$ (Tabela 2). Cerri (1989) e Longo (1999), também observaram que o conteúdo de $\mathrm{C}$ e de $\mathrm{N}$ no solo são variáveis, por estarem concentrados na camada superficial do solo, a qual sofre influência da serapilheira.

$\mathrm{Na}$ comparação das duas coberturas vegetais (floresta primária e cupuaçuzal), não houve diferença significativa no conteúdo de matéria orgânica na CA (Tabela 2). Nye \& Greeland (1964) também constataram que somente com a retirada da floresta e com a implantação de outra cultura, sem que ocorra algum distúrbio nas características físicas, o conteúdo de matéria orgânica no solo não é alterado. Choné et al. (1991) demonstraram que, com a implantação de culturas, especialmente pastagens, o estoque de $\mathrm{C}$ no solo se mantém estável, e em alguns casos pode até superar o conteúdo presente na floresta. Resultados inversos foram obtidos no solo sob cupuaçuzeiro com três anos de idade (Tabela 2). Segundo Demattê (1988), após a remoção da mata e com o cultivo contínuo, o teor de MO nos primeiros centímetros do solo decresce com o aumento da temperatura, as perdas por erosão, a maior atividade biológica, e principalmente com a redução da fonte supridora de resíduos orgânicos.

As relações $\mathrm{C} / \mathrm{N}$ apresentaram comportamentos distintos nas duas cronosseqüências, quando comparadas com os teores de $\mathrm{N}$ e de $\mathrm{MO}$. A relação $\mathrm{C} / \mathrm{N}$ na floresta primária mostrou-se mais estável, com diferenças significativas a partir da camada de $20-40 \mathrm{~cm}$ na $C A$ e na de 40-60 cm na CB, o que não foi observado nas áreas de plantio (Tabela 2). Nestas condições, a formação da MO está em equilíbrio, sofrendo pequenas alterações, o que não foi observado nas áreas de cultivo.

$\mathrm{Na} \mathrm{CB}$, a diferença na relação $\mathrm{C} / \mathrm{N}$ entre as coberturas vegetais foi mais acentuada que na $\mathrm{CA}$, mostrando que a diferenciação no manejo do solo altera significativamente o quociente (Tabela 2). Na média das quatro profundidades, houve aumento de $6,21 \%$ na relação $\mathrm{C} / \mathrm{N}$ na transecção floresta primária/área de cultivo na cronosseqüência $\mathrm{A}$, ao passo que na $\mathrm{CB}$ verificou-se redução de $17,30 \%$.

A diminuição na relação $\mathrm{C} / \mathrm{N}$ no cupuaçuzal da cronosseqüência $\mathrm{B}$ pode estar relacionada ao aumento da população de leguminosas que surgem após o enleiramento, queima do material vegetal e introdução de pastagem. Na floresta primária, o aumento da relação $\mathrm{C} / \mathrm{N}$ e a diminuição da mineralização da matéria orgânica do solo geralmente ocorrem pela alta quantidade de folhas, ramos e galhos, que são de difícil decomposição (Vieira \& Santos, 1987). A relação C/N na camada de 0-10 cm, nas duas cronossequiências, situouse na faixa de 10 a 15 encontradas por Falesi et al. (1969) em Latossolo Amarelo distrófico da região de Manaus, AM (Tabela 2).

A floresta primária apresentou, independentemente da cronosseqüência, semelhança nas quantidades de amônio, nitrato e nitrogênio mineral, havendo diferenças somente entre as áreas de manejo (Tabela 2). Independentemente das cronosseqüências, com exceção do amônio na CA, a concentração de amônio, nitrato e $\mathrm{N}$ mineral foram significativamente maiores nos cupuaçuzais.

No solo sob floresta primária, a concentração de $\mathrm{N}$ nítrico foi sempre menor que a de $\mathrm{N}$ amoniacal, independentemente da camada amostrada, totalizando, na média, 20,0\% do $\mathrm{N}$ mineral. Nas duas áreas sob cultivo, exceto na camada $0-10 \mathrm{~cm}$ da cronossequiência $\mathrm{B}$, as concentrações de nitrato foram maiores, o que demonstra a rápida conversão de $\mathrm{NH}_{4}{ }^{+}$em $\mathrm{NO}_{3}{ }^{-}$. A maior presença de leguminosas e menores valores na relação $\mathrm{C} / \mathrm{N}$ (Malavolta, 1987) nas áreas de cultivo, exceto na camada 10-20 cm da cronossequiência A, devem ter acelerado o processo de nitrificação, que deve ocorrer mais lentamente nas áreas sob floresta. Trabalhos realizados por Piccolo (1994) demonstraram que o amônio foi a forma dominante de $\mathrm{N}$ inorgânico em solo sob floresta.

Ao contrário dos dados de Schroth et al. (2000), obtidos num estudo sobre a variabilidade espacial da mineralização do N, em Latossolo Amarelo distrófico cultivado com dendezeiro, não houve incremento na quantidade de nitrato no perfil do solo, sendo os maiores teores encontrados na camada de $0-10 \mathrm{~cm}$, o mesmo ocorrendo com o amônio (Tabela 2).

O C da biomassa microbiana determinada pelo método de irradiação e extração (IE) e o IRGA, apresentaram correlação significativa (Figura 1). Apesar da significância, a estimativa de $\mathrm{C}-\mathrm{CO}_{2}$ microbiano feita indiretamente com o IRGA revelou valores, na média, 3,45 vezes menores que o IE (Tabela 3 ).

$\mathrm{O}$ conteúdo do $\mathrm{C}$ da biomassa microbiana variou, na média, no método de irradiação e extração (IE) de $708,23 \mathrm{mg} \mathrm{C} \mathrm{kg}^{-1}$ de solo na floresta primária a 
472,66 mg C kg-1 de solo no manejo 1, e no IRGA, de $196,89 \mathrm{mg} \mathrm{C} \mathrm{kg}^{-1}$ de solo na floresta primária a 148,68 mg C kg-1 de solo também no manejo 1 (Tabela 3).

Os valores obtidos nos dois métodos diferiram significativamente entre a floresta primária e a sucessão com cupuaçuzeiro, não havendo diferenças entre os dois tipos de manejo (Tabela 3). A menor taxa de decomposição na floresta primária foi, provavelmente, decorrente da maior relação $\mathrm{C} / \mathrm{N}$ da biomassa microbiana. Altas relações (lignina + polifenóis)/ $\mathrm{N}$ em material vegetal de diferentes espécies florestais indicam baixa capacidade

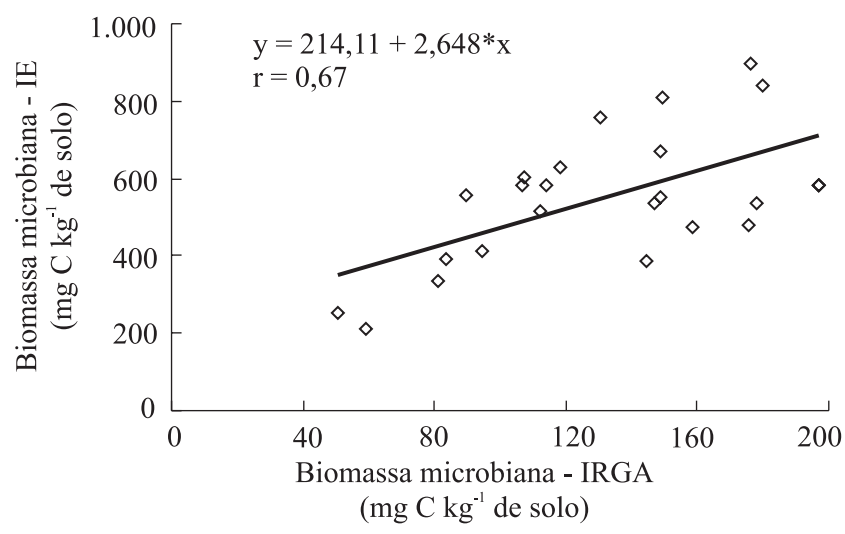

Figura 1. Relação entre a biomassa microbiana de carbono determinada por Infra Red Gas Analyzer (IRGA) e por irradiação extração (IE). * Significativo a 5\% de probabilidade pelo teste F. de mineralização de $\mathrm{N}$, resultando em baixa taxa de decomposição (Gama-Rodrigues et al., 1997).

Cerri et al. (1985), estudando os efeitos da sucessão floresta primária e área de cultivo na qualidade do solo, verificaram a ocorrência de uma dispersão do carbono proveniente da serapilheira, que favoreceu a microbiota do solo. Com o desmatamento e queima do material vegetal, entretanto, ocorrem modificações nas características químicas, físicas e biológicas do solo, provocando, geralmente, considerável inibição da biomassa microbiana.

As amostras sob manejo 2 apresentaram menor atividade respiratória (respiração basal $-2,83 \mu \mathrm{g} \mathrm{g}^{-1} \mathrm{dia}^{-1}$ de $\mathrm{C}-\mathrm{CO}_{2}$ no solo), diferindo estatisticamente da floresta primária e do manejo 1 (Tabela 3). A menor liberação de $\mathrm{CO}_{2}$ nesse ecossistema indica menor atividade biológica, exceto em solos com alto teor de MO totalmente humificada. Este fato está diretamente relacionado com o C da matéria orgânica (Fernandes, 1999). O quociente metabólico $\left(\mathrm{qCO}_{2}\right)$ apresentou menor valor no solo sob floresta. Segundo Balota et al. (1998), a respiração basal por unidade de biomassa microbiana diminui em agroecossitemas mais estáveis, porém, com a substituição da cobertura vegetal ocorre decomposição mais acelerada dos resíduos vegetais, aumentando o quociente metabólico (Ocio \& Brookes, 1990). Tais resultados indicaram relação inversa entre o $\mathrm{qCO}_{2}$ e a biomassa microbiana, ou seja, maiores teores de carbono causam diminuição na atividade metabólica e aumento da biomassa microbiana (Balota et al., 1998).

Tabela 3. Respiração basal, fluxo de $\mathrm{CO}_{2}$, biomassa microbiana obtida por irradiação e extração (IE), biomassa microbiana obtida pelo IRGA, relação $\mathrm{C}_{\min } / \mathrm{C}_{\text {org }}$, quociente metabólico e relação $\mathrm{C} / \mathrm{N}$ da biomassa microbiana na camada de $0-10 \mathrm{~cm}$ de profundidade nas três coberturas vegetais ${ }^{(1)}$.

\begin{tabular}{|c|c|c|c|}
\hline Variável & Floresta primária & Manejo $1^{(2)}$ & Manejo $2^{(3)}$ \\
\hline Respiração basal ( $\mu \mathrm{g} \mathrm{g}^{-1} \mathrm{dia}^{-1}$ de $\mathrm{C}-\mathrm{CO}_{2}$ no solo) & $3,12 \mathrm{a}$ & $3,21 \mathrm{a}$ & $2,83 b$ \\
\hline Fluxo de $\mathrm{CO}_{2}\left(\mathrm{~mL} \mathrm{~min}^{-1}\right)$ & $494,99 a$ & $446,93 a$ & $494,62 a$ \\
\hline Biomassa microbiana (mg C kg-1 de solo) - IE & $708,23 a$ & $472,66 b$ & $535,45 b$ \\
\hline Biomassa microbiana (mg C kg-1 de solo) - IRGA & $196,89 \mathrm{a}$ & $148,68 b$ & $149,77 \mathrm{~b}$ \\
\hline Biomassa microbiana ( $\mathrm{mg} \mathrm{N} \mathrm{kg}^{-1}$ de solo) - IE & $46,82 b$ & $77,34 \mathrm{a}$ & $59,92 \mathrm{a}$ \\
\hline $\mathrm{C}_{\min } / \mathrm{C}_{\mathrm{org}}(\%)$ & $2,22 \mathrm{a}$ & $1,94 \mathrm{a}$ & $2,26 \mathrm{a}$ \\
\hline $\mathrm{qCO}_{2}\left(\mu \mathrm{g} \mathrm{CO}_{2} / \mu \mathrm{g} \mathrm{C}_{\min } \mathrm{h}^{-1}\right) \times 10^{-4}$ & $6,60 \mathrm{~b}$ & $8,99 \mathrm{a}$ & $7,87 \mathrm{a}$ \\
\hline $\mathrm{C} / \mathrm{N}$ da biomassa & $15,13 \mathrm{a}$ & $6,11 \mathrm{c}$ & $8,94 b$ \\
\hline
\end{tabular}

${ }^{(1)}$ Valores seguidos por letras distintas na mesma linha diferem entre si a $5 \%$ de probabilidade pelo teste de Tukey; $\mathrm{C}_{\min } / \mathrm{C}_{\text {org }}$ : biomassa microbiana/ carbono orgânico total; $\mathrm{qCO}_{2}$ (quociente metabólico): respiração basal/carbono microbiano (IRGA). ${ }^{(2)}$ Manejo 1: cupuaçuzal de três anos, cuja implantação não utilizou implementos agrícolas, havendo somente a retirada da madeira, abertura e adubação das covas e plantio. (3)Manejo 2: cupuaçuzal de três anos, instalado sob área de pastagem de Brachiaria humidicula (oito anos), com posterior adubação das covas e plantio.

Pesq. agropec. bras., Brasília, v.39, n.11, p.1103-1110, nov. 2004 


\section{Conclusões}

1. A sucessão floresta primária-pastagem-cupuaçuzal causa diminuição significativa da matéria orgânica e do $\mathrm{C}$ da biomassa microbiana do solo, ao passo que na sucessão floresta primária-cupuaçuzal ocorre diminuição apenas do $\mathrm{C}$ da biomassa microbiana.

2. Independentemente do manejo adotado, o $\mathrm{N}$ da biomassa microbiana da camada de $0-10 \mathrm{~cm}$ aumenta significativamente na área de cupuaçuzal, verificandose o inverso com o $\mathrm{N}$ total.

3. A floresta primária apresenta menor quociente metabólico e maior relação $\mathrm{C} / \mathrm{N}$ da biomassa, o que resulta em menor perda de carbono.

4. A concentração de amônio no solo diminui em profundidades maiores, diferentemente do que ocorre com o nitrato.

\section{Agradecimentos}

Ao $\mathrm{CNPq}$ e à Fapesp, pelo suporte financeiro.

\section{Referências}

ANDERSON, J.P.E.; DOMSCH, K.H. A physiological method for the quantitative measurement of microbial biomass in soils. Soil Biology and Biochemestry, v.10, p.215-221, 1978.

ANDREUX, F.; CERRI, C.C. Current trends in the research on soil changes due to deforestation, burning and cultivation in the Brazilian tropics. Toxocological and Environmental Chemistry, v.20/22, p.275-283, 1989.

BALOTA, E.L.; COLOZZI-FILHO, A.; ANDRADE, D.S.; HUNGRIA, M. Biomassa microbiana e sua atividade em solos sob diferentes sistemas de preparo e sucessão de culturas. Revista Brasileira de Ciência do Solo, v.22, p.641-650, 1998.

CARVALHO, A.F. Caracterização física, química e mineralógica dos solos do Município de Humaitá - AM. 1986. 166p. Tese (Livre docência) - Universidade Estadual Paulista Júlio Mesquita, Botucatu.

CERRI, C.C. Dinâmica da matéria orgânica em solo de pastagem. In: SIMPÓSIO SOBRE ECOSSISTEMA DE PASTAGEM, 1989, Piracicaba. Anais. Piracicaba: Universidade de São Paulo, p.135147, 1989.

CERRI, C.C.; VOLKOFF, B.; EDUARDO, B.P. Efeito do desmatamento sobre a biomassa microbiana em Latossolo Amarelo da Amazônia. Revista Brasileira de Ciência do Solo, v.9, p.1-4, 1985.

CHONÉ, T.; ANDREUX, F.; CORREA, J.C.; VOLKOFF, B.; CERRI, C.C. Changes in organic matter in an Oxisol from the central Amazonian forest during eight years as pastures, determined by ${ }^{13} \mathrm{C}$ isotopic composition. In: BERTHELIN, J. (Ed.). Diversity of environmental biogeochemistry. Amsterdam: Elsevier, 1991.p.397-405.
DEMATTÊ, J.L.I. Manejos de solos ácidos dos trópicos úmidos - Região Amazônica. Campinas: Fundação Cargill, 1988. 215p.

DIEZ, J.A.; POLO, A.; CERRI, C.C.; ANDREUX, F. Efectos comparativos de cultivos intensivos sobre nutrimentos en oxisoles desforestados. Turrialba, v.41, p.150-159, 1991.

EMBRAPA. Centro Nacional de Pesquisa de Solos (Rio de Janeiro, RJ). Manual de métodos de análise de solo. Rio de Janeiro, 1997. 212p.

EMBRAPA. Centro Nacional de Pesquisa de Solos (Rio de Janeiro, RJ). Sistema brasileiro de classificação de solos. Brasília: Embrapa-SPI; Embrapa-CNPS, 1999. 412p.

FALESI, I.C.; CRUZ, E.S.; PEREIRA, F.B.; LOPES, E.C. Os solos da área de Manaus-Itacoatiara. Belém: Ipean, 1969. 116p.

FEARNSIDE, P.M. Deforestation in Brazilian Amazonia: the effect of population and land tenure. Ambio, v.22, p.537-545, 1993.

FEIGL, B.J. Dinâmica da matéria orgânica do solo na sucessão floresta/pastagem na Amazônia (Rondônia). 1994. 68p. Tese (Doutorado) - Centro de Energia Nuclear na Agricultura, Universidade de São Paulo, Piracicaba.

FELLER, C.; FRITCH, E.; POSS, R. Effect de la texture sur le stockage et la dinamique des matièries organiques dans quelques sols ferrugineux et ferralitiques (Afrique de l'ouest, en particulier). Paris: Orstom, 1991. v.26, p.25-36.

FERNANDES, S.A.P. Propriedades do solo na conversão de floresta em pastagem fertilizada e não fertilizada com fósforo na Amazônia (Rondônia). Piracicaba, 1999. 131p. Tese (Doutorado) - Centro de Energia Nuclear na Agricultura, Universidade de São Paulo, Piracicaba.

FERREIRA, A.S.; CAMARGO, F.A.O.; VIDOR, C. Utilização de microondas na avaliação da biomassa microbiana do solo. Revista Brasileira de Ciência do Solo, v.23, p.991-996, 1999.

GAMA-RODRIGUES, E.F.; GAMA-RODRIGUES, A.C.; BARROS, N.F. Biomassa microbiana de carbono e de nitrogênio de solos sob diferentes coberturas florestais. Revista Brasileira de Ciência do Solo, v.21, p.361-366, 1997.

JENKINSON, D.S. Determination of microbial biomass carbon and nitrogen in soil. In: WILSON, J.B. (Ed.). Advances in nitrogen cycling. Wallingford: CAB International, 1988. p.368-386.

JENKINSON, D.S.; LADD, J.N. Microbial biomass in soil measurement and turnover. In: PAUL, E.A.; LADD, J.N. (Ed.). Soil biochemistry. New York: Dekker, 1981. v.5.

JENNY, H. Factors of soil formation: A system of quantitative pedology. New York: CAB International, 1941. 286p.

LONGO, R.M. Modificações em parâmetros físicos e químicos de latossolos argilosos decorrentes da substituição de floresta e de cerrado por pastagens. 1999. 102p. Tese (Doutorado) Universidade de Campinas, Campinas.

LUIZÃO, R.C.C.; BONDE, T.A.; ROSSWALL, T. Seasonal variation of soil microbial biomass: The effects of clear-felling a tropical rainforest and establishment of pasture in the Central Amazon. Soil Biology and Biochemistry, v.24, p.805-813, 1992.

MALAVOLTA, E. Fertilidade dos solos da Amazônia. In: VIEIRA, L.S.; SANTOS, P.C.T.C. (Ed.). Amazônia: seus solos e outros recursos naturais. São Paulo: Agronômica Ceres, 1987. p.374-416. 
MARTINS, P.F. da S.; CERRI, C.C.; VOLKOFF, B.; ANDREUX, F.; CHAUVEL, A. Consequences of clearing and tillage on the soil of a natural Amazonian ecosystem. Forest Ecology and Management, v.38, p.273-282, 1991.

MEHLICH, A. Mehlich 3 soil test extractant; a modification of Mehlich 2 extractant. Communications in Soil Science and Plant Analysis, v.15, p.1409-1416, 1984.

NYE, P.H.; GREELAND, D.J. Changes in the soil after clearing tropical forest. Plant and Soil. v.21, p.101-107, 1964.

OCIO, J.A.; BROOKES, P.C. An evaluation of methods for measuring the microbial biomass in soils following recent additions of wheat straw and characterization of the biomass that develops. Soil Biology and Biochemistry, v.22, p.685-694, 1990.

PICCOLO, M.C. Comportamento do nitrogênio do solo em cronosseqüiências de floresta-pastagem em Rondônia. 1994. 69p. Tese (Doutorado) - Centro de Energia Nuclear na Agricultura, Universidade de São Paulo, Piracicaba.

PIMENTEL-GOMES, F. Curso de Estatística Experimental. Piracicaba: Nobel, 1990. 468p.
RAIJ, B. van; ANDRADE, J.C.; CANTARELLA, H.; QUAGGIO, J.A. Análise química para avaliação da fertilidade de solos tropicais. Campinas: Instituto Agronômico, 2001. 284p.

SANCHEZ, P.A.; BANDY, D.E.; VILLACHICA, J.H.; NICHOLAIDES, J.J. Amazon basin soils: Management for continuous crop production. Science, v.216, p.821-827, 1982.

SCHROTH, G.; RODRIGUES, M.R.L.; D’ANGELO, S.A. Spatial patterns of nitrogen mineralization, fertilizer distribution and roots explain nitrate leaching from mature Amazonian oil palm plantation. Soil Use and Management, v.16, p.222-229, 2000.

SPARLING, G.P.; ROSS, D.J. Biochemical methods to estimate soil microbial biomass: current development and applications. In: MULUNGOY, K.; MERCKX, R. (Ed.). Soil organic matter dynamics and sustainability of tropical agriculture. Leuven: WilleySayce, 1993. p.21-37.

VIEIRA, L.S.; SANTOS, P.C.T.C. Amazônia: seus solos e outros recursos naturais. São Paulo: Ceres, 1987. 416p.

Recebido em 25 de maio de 2004 e aprovado em 29 de setembro de 2004 